\title{
CORRECTION
}

https://doi.org/10.1038/s41586-018-0663-4

\section{Author Correction: Phosphocode- dependent functional dichotomy of a common co-receptor in plant signalling}

Artemis Perraki, Thomas A. DeFalco, Paul Derbyshire, Julian Avila, David Séré, Jan Sklenar, Xingyun Qi,

Lena Stransfeld, Benjamin Schwessinger, Yasuhiro Kadota, Alberto P. Macho, Shushu Jiang, Daniel Couto, Keiko U. Torii, Frank L. H. Menke \& Cyril Zipfel

Correction to: Nature https://www.nature.com/articles/s41586-0180471-x, published online 03 September 2018.

In Extended Data Fig. 5d of this Letter, the blots for anti-pS612 and anti-BAK1 were inadvertently duplicated, owing to a copy and paste error during figure preparation. (The original blots presented in Supplementary Fig. 1 were correct.) The original figure has been corrected online, and see Fig. 1 to this Amendment for the old, incorrect Extended Data Fig. 5d, for transparency.

Original Extended Data Fig. 5d

d

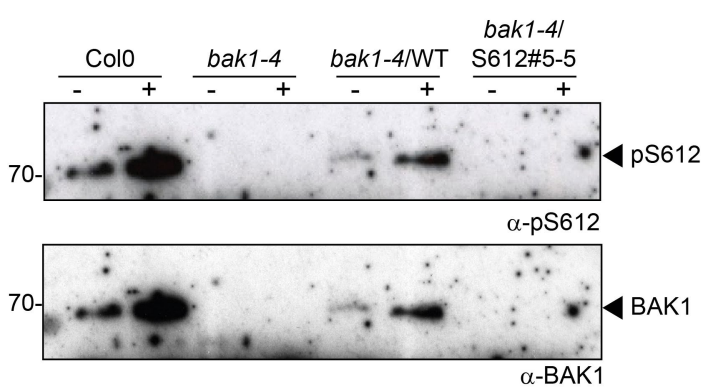

Corrected Extended Data Fig. 5d

d

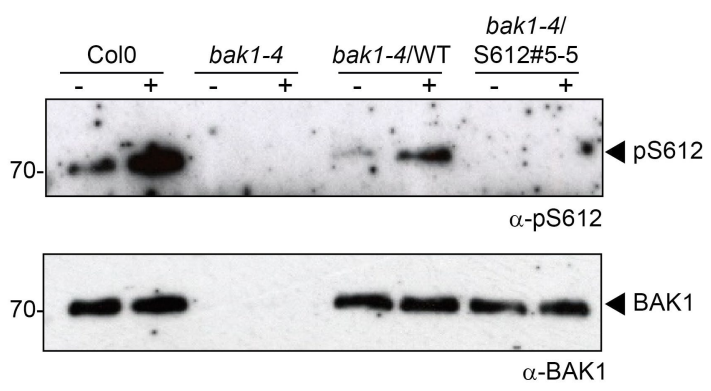

Fig. 1 | This figure displays the corrected panels and the incorrect published panels from Extended Data Fig. 5d of the original Letter. 